TITLE:

\title{
Equivalent-Circuit Model for Meta- Atoms Consisting of Wired Metallic Spheres
}

\section{AUTHOR(S):}

HISAKADO, Takashi; YOSHIDA, Keisuke;

MATSUSHIMA, Tohlu; WADA, Osami

\section{CITATION:}

HISAKADO, Takashi ...[et al]. Equivalent-Circuit Model for Meta-Atoms Consisting of Wired Metallic Spheres. IEICE Transactions on Electronics 2017, E100.C(3): 305-312

ISSUE DATE:

2017-03

URL:

http://hdl.handle.net/2433/252316

RIGHT:

(C) 2017 The Institute of Electronics, Information and Communication Engineers 
Equivalent-Circuit Model for Meta-Atoms Consisting of Wired Metallic Spheres

\author{
Takashi HISAKADO $^{\dagger \text { a) }}$, Member, Keisuke YOSHIDA $^{\dagger}$, Student Member, Tohlu MATSUSHIMA ${ }^{\dagger}$, Member, $^{\dagger}$ \\ and Osami WADA ${ }^{\dagger}$, Fellow
}

SUMMARY An equivalent-circuit model is an effective tool for the analysis and design of metamaterials. This paper describes a systematic and theoretical method for the circuit modeling of meta-atoms. We focus on the structures of wired metallic spheres and propose a method for deriving a sophisticated equivalent circuit that has the same topology as the wires using the partial element equivalent circuit (PEEC) method. Our model contains the effect of external electromagnetic coupling: excitation by an external field modeled by voltage sources and radiation modeled by the radiation resistances for each mode. The equivalent-circuit model provides the characteristics of meta-atoms such as the resonant frequencies and the resonant modes induced by the current distribution in the wires by an external excitation. Although the model is obtained by a very coarse discretization, it provides a good agreement with an electromagnetic simulation.

key words: metamaterial, meta-atom, equivalent circuit, wired metallic spheres, PEEC

\section{Introduction}

There has been growing interest in the design and synthesis of meta-atoms and metamaterials. To properly design them, it is very important to elucidate the electromagnetic nature produced by the geometric complexity of structured conductors. An equivalent-circuit model is a powerful tool for describing electromagnetic phenomena in a physically intuitive manner [1]-[7]. However, the equivalent-circuit models for meta-atoms and metamaterials are usually heuristic, and studies on formal constructions from Maxwell's equations to the equivalent-circuit model are limited. We consider a systematic method for deriving a sophisticated equivalent-circuit model using the partial equivalent electric circuit (PEEC) method [8]-[12].

The PEEC method is an integral equation approach, which is the so-called method of moments [13] and was first introduced in [8]. The method systematically provides equivalent-circuit models from Maxwell's equations and is useful for interconnection modeling in VLSI design. However, the conditions for the basis functions require small meshes to model the general metallic structures, and the number of circuit elements becomes very large, making the model very complicated for design. In order to derive a simple equivalent-circuit model, we introduce a structure of wired metallic spheres [14]. An example of the structure is

Manuscript received August 31, 2016.

Manuscript revised November 9, 2016.

${ }^{\dagger}$ The authors are with Department of Electrical Engineering, Kyoto University, Kyoto-shi, 615-8510 Japan.

a) E-mail: hisakado@kuee.kyoto-u.ac.jp

DOI: 10.1587/transele.E100.C.305 shown in Fig. 1.

When the size of each component in the structure is sufficiently smaller than the wavelength of the incident field, and the radii of the spheres are sufficiently smaller than the lattice constant, we assume that induced currents exist only at the center of thin wires with a uniform distribution and that the charges exist only at the centers of spheres, except for the estimation of self-interactions such as selfinductances and self-capacitances. On the basis of this approximation, we derive a very simple equivalent-circuit model that has the same topology as the wires. That is, the wires and spheres correspond to the branches and nodes in the equivalent-circuit model, respectively.

This paper provides a theoretical and systematic method for deriving a simple equivalent circuit model from Maxwell's equation with sufficient flexibility for different geometric parameters. The simple model enables us to estimate the electromagnetic characteristics of the meta-atoms and provides physical insights. The proposed circuit equation includes not only the electromagnetic couplings between the wires and between the spheres in the structure, but also external coupling, i.e., excitation by an external field and radiation. The model provides characteristics of meta-atoms such as the resonant frequencies and resonant modes induced in the wires due to an external excitation. Although the model is obtained by a very coarse discretization, it agrees sufficiently with electromagnetic simulation.

This paper is organized as follows. In Sect. 2, we present a systematic method for deriving the circuit equation of the wired metallic spheres from Maxwell's equations. In Sect. 3, we analyze the resonance frequency using a simple example of the model. In Sect. 4, we propose a method for modeling the radiation by the radiation resistance, which is

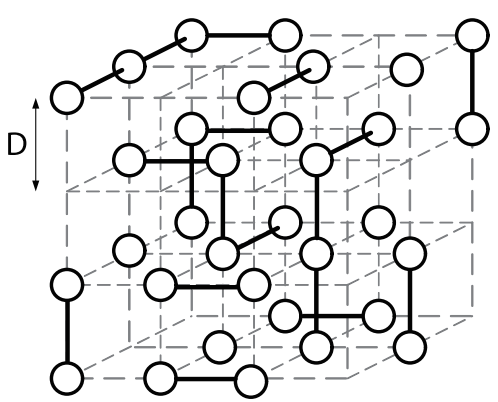

Fig. 1 Metamaterial with wired metallic spheres. 
essential for the metamaterial model. In Sect. 5, we compare the structures for ten examples of meta-atoms and present a comparison with an electromagnetic analysis.

\section{Derivation of the Circuit Equation}

\subsection{Electric-Field Integral Equation}

To derive the circuit equation in the Lorenz gauge, we start the derivation from the Maxwell's equations expressed as a set of wave equations

$$
\begin{aligned}
& \square^{2} \boldsymbol{A}=\mu_{0} \boldsymbol{J}, \\
& \square^{2} \phi=\frac{\rho}{\varepsilon_{0}},
\end{aligned}
$$

where $\square^{2}$ is the d'Alembert operator; $\boldsymbol{A}$ and $\phi$ denote the vector and scalar potentials, respectively; $\boldsymbol{J}$ and $\rho$ denote the current and charge densities, respectively; and $\epsilon_{0}$ and $\mu_{0}$ are the vacuum permittivity and permeability, respectively. Equations (1) and (2) are coupled by the continuity equation

$$
\boldsymbol{\nabla} \cdot \boldsymbol{J}+\frac{\partial \rho}{\partial t}=0
$$

which expresses the conservation of charge.

The Green's function of the wave equation is given by

$$
G\left(\boldsymbol{r}, t, \boldsymbol{r}^{\prime}, t^{\prime}\right) \equiv \frac{\delta\left(t-t^{\prime}-\frac{\left|\boldsymbol{r}-\boldsymbol{r}^{\prime}\right|}{\mathrm{c}}\right)}{4 \pi\left|\boldsymbol{r}-\boldsymbol{r}^{\prime}\right|},
$$

where $\mathrm{c}$ is the speed of light. Then, the potentials $\boldsymbol{A}$ and $\phi$ in Eqs. (1) and (2) are written by

$$
\begin{aligned}
& \boldsymbol{A}(\boldsymbol{r}, t)=\mu_{0} \iint G\left(\boldsymbol{r}, t, \boldsymbol{r}^{\prime}, t^{\prime}\right) \boldsymbol{J}\left(\boldsymbol{r}^{\prime}, t^{\prime}\right) \mathrm{d}^{3} \boldsymbol{r}^{\prime} \mathrm{d} t^{\prime}, \\
& \phi(\boldsymbol{r}, t)=\frac{1}{\varepsilon_{0}} \iint G\left(\boldsymbol{r}, t, \boldsymbol{r}^{\prime}, t^{\prime}\right) \rho\left(\boldsymbol{r}^{\prime}, t^{\prime}\right) \mathrm{d}^{3} \boldsymbol{r}^{\prime} \mathrm{d} t^{\prime} .
\end{aligned}
$$

Because the electric field is given by

$$
\boldsymbol{E}=-\frac{\partial \boldsymbol{A}}{\partial t}-\boldsymbol{\nabla} \phi
$$

the boundary condition in the tangential direction on the surface of a perfect conductor is expressed as

$$
\begin{aligned}
& \mu_{0} \frac{\partial}{\partial t} \iint G\left(\boldsymbol{r}, t, \boldsymbol{r}^{\prime}, t\right) \boldsymbol{J}\left(\boldsymbol{r}^{\prime}, t^{\prime}\right) \mathrm{d} \boldsymbol{r}^{\prime} \mathrm{d} t^{\prime} \\
& +\frac{1}{\epsilon_{0}} \boldsymbol{\nabla} \iint G\left(\boldsymbol{r}, t, \boldsymbol{r}^{\prime}, t\right) \rho\left(\boldsymbol{r}^{\prime}, t^{\prime}\right) \mathrm{d} \boldsymbol{r}^{\prime} \mathrm{d} t^{\prime}=\boldsymbol{E}^{\mathrm{E}}(\boldsymbol{r}, t),
\end{aligned}
$$

where $\boldsymbol{E}^{\mathrm{E}}$ is the incident electric field. We formulate the circuit equation using Eqs. (3) and (8).

\subsection{Spatial Discretization}

We assume that the structure consists of a perfect conductor and that the lattice constant $D$ in Fig. 1 is sufficiently smaller than the wavelength $\lambda$ of the incident field. For simplicity,

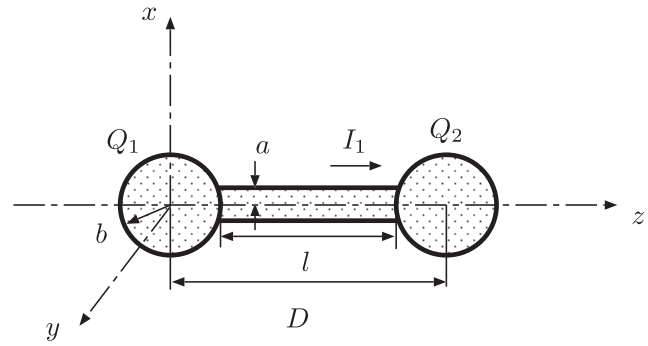

Fig. 2 Parameters for a simple structure of wired spheres.

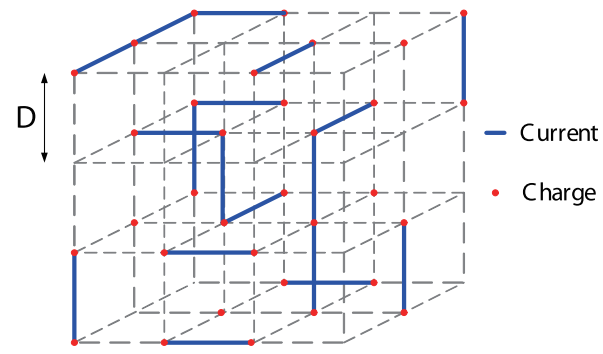

Fig. 3 Model with line currents and a point charge.

we explain the spatial discretization of Eqs. (3) and (8) using a simple structur of the meta-atom shown in Fig. 2. The radius of the wire, the radius of the spheres, and the length of the wire are denoted by $a, b$, and $l=D-2 b$, respectively. We assume that these parameters satisfy $a \ll b \ll$ $l \ll \lambda$ and approximate that the charges exist at the centers of the spheres and current exists along the central axis of the wire with a uniform distribution, except for the estimation of self-interactions such as the self-inductance and self-capacitance. Then, the model of the structure in Fig. 1 is represented by Fig. 3, and we formulate a circuit equation in which the wires and spheres correspond to the circuit branches and nodes, respectively. On the other hand, the self-interactions are estimated with the parameters $a, b$, and $l$.

We introduce a very coarse discretization for Eqs. (3) and (8), in which one wire in the structure corresponds to one branch in the circuit in order to derive the very simple model. Although the model includes errors caused by approximating the uniform distribution of the currents on the wires and the charges on the spheres, the condition is approximately satisfied for the structure with $a \ll b \ll l \ll \lambda$. As a result, the resonant frequencies of the proposed model and that of the electromagnetic simulation shown in later sections differ by several percent.

For example, the basis for the wire current in Fig. 2 is described by

$$
\boldsymbol{\Psi}_{1}(\boldsymbol{r}) \equiv \delta(x) \delta(y)\{h(z)-h(z-D)\} \hat{z},
$$

where $\hat{z}$ is a unit vector in the $z$ direction, $h(z)$ is the Heaviside unit-step function, and $\delta$ is the Dirac delta function. The basis for the charges in the two spheres in Fig. 2 is represented by

$$
\Phi_{1}(\boldsymbol{r}) \equiv \delta(x) \delta(y) \delta(z), \quad \Phi_{2}(\boldsymbol{r}) \equiv \delta(x) \delta(y) \delta(z-D) .
$$


Using such bases for the currents and charges, we express the current density $\boldsymbol{J}(\boldsymbol{r}, t)$ and the charge density $\rho(\boldsymbol{r}, t)$ as

$$
\begin{gathered}
\boldsymbol{J}(\boldsymbol{r}, t)=\sum_{n} I_{n}(t) \boldsymbol{\Psi}_{n}(\boldsymbol{r}), \\
\rho(\boldsymbol{r}, t)=\sum_{m} Q_{m}(t) \Phi_{m}(\boldsymbol{r}),
\end{gathered}
$$

where $I_{n}$ is the current in the $n$-th wire, and $Q_{m}$ is the charge on the $m$-th sphere. Here, the bases $\boldsymbol{\Psi}_{n}(\boldsymbol{r})$ and $\Phi_{m}(\boldsymbol{r})$ satisfy

$$
\nabla \cdot \Psi_{n}(\boldsymbol{r})=\sum_{m} U_{m n} \Phi_{m}(\boldsymbol{r}),
$$

where $U_{m n}$ is the incidence matrix

$$
U_{m n}= \begin{cases}1 & \text { if } \boldsymbol{\Psi}_{n}(\boldsymbol{r}) \text { leaves } \Phi_{m}(\boldsymbol{r}) \\ -1 & \text { if } \boldsymbol{\Psi}_{n}(\boldsymbol{r}) \text { enters } \Phi_{m}(\boldsymbol{r}) \\ 0 & \text { otherwise, }\end{cases}
$$

which represents the topology of the wired spheres.

By substituting Eqs. (11) and (12) into Eq. (3) and using Eq. (13), we obtain

$$
\sum_{m}\left\{\sum_{n} U_{m n} I_{n}(t)+\frac{\partial}{\partial t} Q_{m}(t)\right\} \Phi_{m}(\boldsymbol{r})=0 .
$$

Then, the conservation of charge on the sphere is rewritten by the relationship between the currents $I_{n}$ and the charges $Q_{m}$ at the nodes

$$
\sum_{n} U_{m n} I_{n}(t)+\frac{\partial}{\partial t} Q_{m}(t)=0 .
$$

2.3 Formulation of the Boundary Condition on the Wire Branches

In order to apply the boundary condition in Eq. (8) to the wire branches, we use the current basis function $\boldsymbol{\Psi}_{n}(\boldsymbol{r})$ for the test function $\boldsymbol{\Psi}_{n}^{\mathrm{W}}(\boldsymbol{r})$, except for the self-interactions. The test function $\boldsymbol{\Psi}_{1}^{\mathrm{S}}(\boldsymbol{r})$ for the self-interactions is defined on the surface of the wire, e.g.,

$$
\Psi_{1}^{\mathrm{S}}(\boldsymbol{r}) \equiv \delta(x-a) \delta(y)\{h(z-b)-h(z-D+b)\} \hat{z},
$$

for Fig. 2 becaues the thin wire is dominant in the selfinductance by the relation $a \ll b$. By substituting Eqs. (11) and (12) into Eq. (8) and using the test function $\boldsymbol{\Psi}_{n}^{\mathrm{W}}(\boldsymbol{r})$, we rewrite Eq. (8) as

$$
\begin{aligned}
& \int \Psi_{n}^{\mathrm{W}}(\boldsymbol{r}) \mu_{0} \frac{\partial}{\partial t} \iint G\left(\boldsymbol{r}, t, \boldsymbol{r}^{\prime}, t^{\prime}\right) \sum_{n^{\prime}} I_{n^{\prime}}\left(t^{\prime}\right) \Psi_{n^{\prime}}\left(\boldsymbol{r}^{\prime}\right) \mathrm{d} \boldsymbol{r}^{\prime} \mathrm{d} t^{\prime} \mathrm{d} \boldsymbol{r} \\
& +\int \Psi_{n}^{\mathrm{W}}(\boldsymbol{r}) \frac{1}{\epsilon_{0}} \nabla \iint G\left(\boldsymbol{r}, t, \boldsymbol{r}^{\prime}, t^{\prime}\right) \sum_{m^{\prime}} Q_{m^{\prime}}\left(t^{\prime}\right) \Phi_{m^{\prime}}\left(\boldsymbol{r}^{\prime}\right) \mathrm{d} \boldsymbol{r}^{\prime} \mathrm{d} t^{\prime} \mathrm{d} \boldsymbol{r} \\
& =\int \Psi_{n}^{\mathrm{W}}(\boldsymbol{r}) \boldsymbol{E}^{\mathrm{E}}(\boldsymbol{r}, t) \mathrm{d} \boldsymbol{r} .
\end{aligned}
$$

Here, the test function is summarized as

$$
\boldsymbol{\Psi}_{n}^{\mathrm{W}}(\boldsymbol{r})= \begin{cases}\boldsymbol{\Psi}_{n}^{\mathrm{S}}(\boldsymbol{r}) & \left(n=n^{\prime}\right) \\ \boldsymbol{\Psi}_{n}(\boldsymbol{r}) & \left(n \neq n^{\prime}\right) .\end{cases}
$$

Further, in order to simplify Eq. (18), we introduce the function $\Phi_{m}^{\mathrm{S}}(\boldsymbol{r})$ that satisfies the relationship in Eq. (13) for the test function $\Psi_{n}^{\mathrm{W}}(\boldsymbol{r})$, e.g.,

$$
\begin{aligned}
& \Phi_{1}^{\mathrm{S}}(\boldsymbol{r}) \equiv \delta(x-a) \delta(y) \delta(z-b), \\
& \Phi_{2}^{\mathrm{S}}(\boldsymbol{r}) \equiv \delta(x-a) \delta(y) \delta(z-D+b),
\end{aligned}
$$

for the test function in Eq. (17) in Fig. 2. Then, Eq. (18) is expressed as

$$
\begin{aligned}
& \sum_{n^{\prime}} \frac{\partial}{\partial t} \int \bar{L}_{n n^{\prime}}\left(t, t^{\prime}\right) I_{n^{\prime}}\left(t^{\prime}\right) \mathrm{d} t^{\prime} \\
& +\sum_{m^{\prime}} \int \bar{S}_{n m^{\prime}}\left(t, t^{\prime}\right) Q_{m^{\prime}}\left(t^{\prime}\right) \mathrm{d} t^{\prime}=-V_{n}^{\mathrm{E}}(t),
\end{aligned}
$$

where

$$
\begin{aligned}
& \bar{L}_{n n^{\prime}}\left(t, t^{\prime}\right) \equiv \mu_{0} \int_{V} \int_{V^{\prime}} \Psi_{n}^{\mathrm{W}}(\boldsymbol{r}) G\left(\boldsymbol{r}, t, \boldsymbol{r}^{\prime}, t^{\prime}\right) \Psi_{n^{\prime}}\left(\boldsymbol{r}^{\prime}\right) \mathrm{d}^{3} \boldsymbol{r}^{\prime} \mathrm{d}^{3} \boldsymbol{r}, \\
& \bar{S}_{n m^{\prime}}\left(t, t^{\prime}\right) \\
& \equiv \frac{1}{\varepsilon_{0}} \int_{V} \boldsymbol{\Psi}_{n}^{\mathrm{W}}(\boldsymbol{r}) \cdot \nabla \int_{V^{\prime}} G\left(\boldsymbol{r}, t, \boldsymbol{r}^{\prime}, t^{\prime}\right) \Phi_{m^{\prime}}\left(\boldsymbol{r}^{\prime}\right) \mathrm{d}^{3} \boldsymbol{r}^{\prime} \mathrm{d}^{3} \boldsymbol{r} \\
& =-\frac{1}{\varepsilon_{0}} \int_{V} \int_{V^{\prime}}\left\{\nabla \cdot \Psi_{n}^{\mathrm{W}}(\boldsymbol{r})\right\} G\left(\boldsymbol{r}, t, \boldsymbol{r}^{\prime}, t^{\prime}\right) \Phi_{m^{\prime}}\left(\boldsymbol{r}^{\prime}\right) \mathrm{d}^{3} \boldsymbol{r}^{\prime} \mathrm{d}^{3} \boldsymbol{r} \\
& =-\sum_{m} U_{m n} \frac{1}{\varepsilon_{0}} \int_{V} \int_{V^{\prime}} \Phi_{m}^{\mathrm{W}}(\boldsymbol{r}) G\left(\boldsymbol{r}, t, \boldsymbol{r}^{\prime}, t^{\prime}\right) \Phi_{m^{\prime}}\left(\boldsymbol{r}^{\prime}\right) \mathrm{d}^{3} \boldsymbol{r}^{\prime} \mathrm{d}^{3} \boldsymbol{r}, \\
& V_{n}^{\mathrm{E}}(t) \equiv-\int_{V} \boldsymbol{\Psi}_{n}^{\mathrm{W}}(\boldsymbol{r}) \boldsymbol{E}_{n}^{\mathrm{E}}(\boldsymbol{r}, t) \mathrm{d}^{3} \boldsymbol{r} .
\end{aligned}
$$

Here, the function $\Phi_{m}^{\mathrm{W}}(\boldsymbol{r})$ is summarized as

$$
\Phi_{m}^{\mathrm{W}}(\boldsymbol{r})= \begin{cases}\Phi_{m}^{\mathrm{S}}(\boldsymbol{r}) & \left(m=m^{\prime}\right) \\ \Phi_{m}(\boldsymbol{r}) & \left(m \neq m^{\prime}\right) .\end{cases}
$$

After introducing the potential coefficient matrix defined by $\bar{P}_{m m^{\prime}}\left(t, t^{\prime}\right) \equiv \frac{1}{\varepsilon_{0}} \int_{V} \int_{V^{\prime}} \Phi_{m}^{\mathrm{W}}(\boldsymbol{r}) G\left(\boldsymbol{r}, t, \boldsymbol{r}^{\prime}, t^{\prime}\right) \Phi_{m^{\prime}}\left(\boldsymbol{r}^{\prime}\right) \mathrm{d}^{3} \boldsymbol{r}^{\prime} \mathrm{d}^{3} \boldsymbol{r}$

to Eq. (22), we obtain the discretized boundary condition

$$
\begin{aligned}
& \sum_{n^{\prime}} \frac{\partial}{\partial t} \int \bar{L}_{n n^{\prime}}\left(t, t^{\prime}\right) I_{n^{\prime}}\left(t^{\prime}\right) \mathrm{d} t^{\prime} \\
& -\sum_{m^{\prime}} \sum_{m} \int U_{m n} \bar{P}_{m m^{\prime}}\left(t, t^{\prime}\right) Q_{m^{\prime}}\left(t^{\prime}\right) \mathrm{d} t^{\prime}=-V_{n}^{\mathrm{E}}(t) .
\end{aligned}
$$

\subsection{Neglecting Retardation}

For simplicity, if we neglect the retardation in the Green's function in Eq. (4), we approximate Eq. (4) as

$$
G\left(\boldsymbol{r}, t, \boldsymbol{r}^{\prime}, t^{\prime}\right) \equiv \frac{\delta\left(t-t^{\prime}\right)}{4 \pi\left|\boldsymbol{r}-\boldsymbol{r}^{\prime}\right|},
$$

resulting in 


$$
\boldsymbol{L} \frac{\mathrm{d}}{\mathrm{d} t} \boldsymbol{I}-\boldsymbol{U}^{T} \boldsymbol{P} \boldsymbol{Q}=-\boldsymbol{V}^{\mathrm{E}},
$$

where $\boldsymbol{I}$ and $\boldsymbol{Q}$ are the current and charge vectors, respectively; $\boldsymbol{U}$ is the incidence matrix; and ${ }^{T}$ indicates the matrix transpose operation. The inductance matrix $\boldsymbol{L}$ and the potential coefficient matrix $\boldsymbol{P}$ are defined by

$$
\begin{aligned}
L_{n n^{\prime}} & \equiv \mu_{0} \int_{V} \int_{V^{\prime}} \Psi_{n}^{\mathrm{W}}(\boldsymbol{r}) \frac{1}{4 \pi\left|\boldsymbol{r}-\boldsymbol{r}^{\prime}\right|} \Psi_{n^{\prime}}\left(\boldsymbol{r}^{\prime}\right) \mathrm{d}^{3} \boldsymbol{r}^{\prime} \mathrm{d}^{3} \boldsymbol{r}, \\
P_{m m^{\prime}} & \equiv \frac{1}{\varepsilon_{0}} \int_{V} \int_{V^{\prime}} \Phi_{m}^{\mathrm{W}}(\boldsymbol{r}) \frac{1}{4 \pi\left|\boldsymbol{r}-\boldsymbol{r}^{\prime}\right|} \Phi_{m^{\prime}}\left(\boldsymbol{r}^{\prime}\right) \mathrm{d}^{3} \boldsymbol{r}^{\prime} \mathrm{d}^{3} \boldsymbol{r},
\end{aligned}
$$

respectively. Equations (16) and (28) are the equivalentcircuit equations with charges and currents. Further, by eliminating $\boldsymbol{Q}$ in Eq. (28) with Eq. (16), we obtain the equivalent-circuit equation for the current $I$ as

$$
\frac{\mathrm{d}^{2}}{\mathrm{~d} t^{2}} \boldsymbol{I}+\boldsymbol{L}^{-1} \boldsymbol{U}^{T} \boldsymbol{P} \boldsymbol{U} \boldsymbol{I}=-\boldsymbol{L}^{-1} \frac{\mathrm{d}}{\mathrm{d} t} \boldsymbol{V}^{\mathrm{E}} .
$$

Because this equivalent-circuit equation is represented by the incidence matrix $\boldsymbol{U}$, the circuit model has the same topology as the structure of the wired spheres.

\section{Resonant Frequencies and Modes}

\subsection{Resonant Frequency}

In the frequency domain, Eq. (31) is expressed as

$$
\left(-\omega^{2} \mathbf{1}+\boldsymbol{L}^{-1} \boldsymbol{U}^{T} \boldsymbol{P} \boldsymbol{U}\right) \hat{\boldsymbol{I}}(\omega)=-\mathrm{j} \omega \boldsymbol{L}^{-1} \hat{\boldsymbol{V}}^{\mathrm{E}}(\omega),
$$

where $\omega$ denotes the angular frequency, and $\mathbf{1}$ is an identity matrix. The eigenvalues of the matrix $\boldsymbol{L}^{-1} \boldsymbol{U}^{T} \boldsymbol{P} \boldsymbol{U}$ represent the resonant angular frequencies of wired metallic spheres.

We show an example of the resonant frequency using the simple structure shown in Fig. 2. Although the example has only one wire, it provides an estimate in borderline case of the condition $l \ll \lambda$ between the wire length $l$ and the wavelength $\lambda$ of the incident wave on the resonant frequency. In this case, Eq. (32) is an equation with one variable $I_{1}$, and the matrix $\boldsymbol{L}^{-1} \boldsymbol{U}^{T} \boldsymbol{P} \boldsymbol{U}$ is expressed as

$$
\boldsymbol{L}^{-1} \boldsymbol{U}^{T} \boldsymbol{P} \boldsymbol{U}=\frac{1}{L_{\mathrm{s}}}[1,-1]\left[\begin{array}{cc}
P_{\mathrm{s}} & P_{\mathrm{m}} \\
P_{\mathrm{m}} & P_{\mathrm{s}}
\end{array}\right]\left[\begin{array}{c}
1 \\
-1
\end{array}\right],
$$

where

$$
\begin{aligned}
& L_{\mathrm{s}}=\frac{\mu_{0}}{4 \pi}\left\{l\left(\log \frac{D^{2}-l^{2}}{a^{2}}-1\right)+D\left(\log \frac{D+l}{D-l}-1\right)\right\}, \\
& P_{\mathrm{S}}=\frac{1}{4 \pi \epsilon_{0} b}, \quad P_{\mathrm{m}}=\frac{1}{4 \pi \epsilon_{0} D} .
\end{aligned}
$$

First, we fix the wire and sphere radii at constant values of $a=0.01 \mathrm{~mm}$ and $b=1 \mathrm{~mm}$, respectively, and calculate the resonance frequencies as a function of the wire length $l$ using the equivalent-circuit model. The results are shown in Fig. 4, and the function is compared with the results from

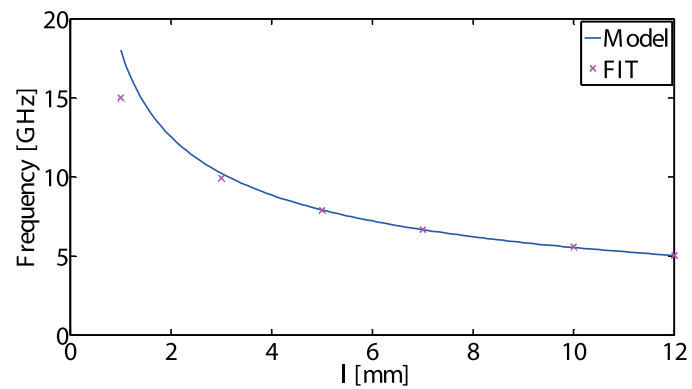

Fig. 4 Resonance frequencies as a function of the wire length $l$. FIT indicates the results from an electromagnetic simulation with a finite integration technique calculated by CST MW SUITE.

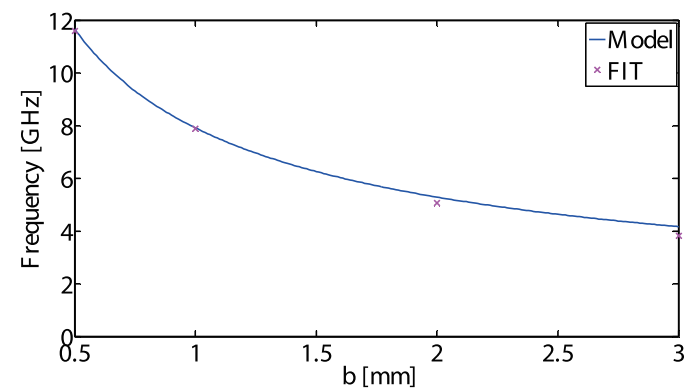

Fig.5 Resonance frequencies as a function of the sphere radius $b$.

an electromagnetic simulation that uses a finite integration technique (FIT) by CST MW SUITE. Although the time required for calculation for every dot in the electromagnetic simulation is greater than one hour, it is noteworthy that the result of the proposed model is obtained only by manual calculation from Eq. (33). In the region of short $l$, there is a relative difference because the condition $b \ll l$ is not satisfied. However, the relative difference between the results is small for the rest of the range of $l$.

Second, we fix the wire radius and length at constant values of $a=0.01 \mathrm{~mm}$ and $l=5 \mathrm{~mm}$, respectively, and calculate the resonance frequencies as a function of the sphere radius $b$. The results for the equivalent-circuit model and FIT are shown in Fig. 5. The relative difference between the results is small, except in the region of large $b$, where the condition $b \ll l$ is not satisfied.

Third, we fix the wire length and sphere radius at constant values of $l=5 \mathrm{~mm}$ and $b=1 \mathrm{~mm}$, respectively, and calculate the resonance frequencies as a function of the wire radius $a$. The results in Fig. 6 show that the relative difference is small; however, the difference becomes larger as $a$ increases. This difference is explained by the requirement $a \ll b$.

\subsection{Resonant Modes}

Using the eigenvectors of the matrix $\boldsymbol{L}^{-1} \boldsymbol{U}^{T} \boldsymbol{P} \boldsymbol{U}$, we obtain the diagonalization of the matrix as

$$
\left(-\omega^{2} \mathbf{1}+\boldsymbol{K}^{-1} \boldsymbol{L}^{-1} \boldsymbol{U}^{T} \boldsymbol{P} \boldsymbol{U} \boldsymbol{K}\right) \boldsymbol{K}^{-1} \hat{\boldsymbol{I}}=-\mathrm{j} \omega \boldsymbol{K}^{-1} \boldsymbol{L}^{-1} \hat{\boldsymbol{V}}^{\mathrm{E}},
$$




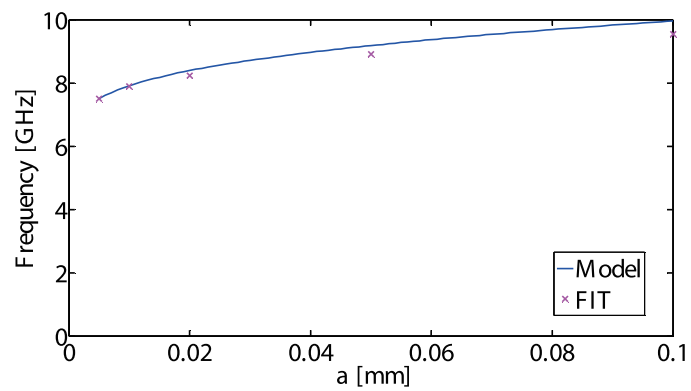

Fig. 6 Resonance frequencies as a function of the wire radius $a$.

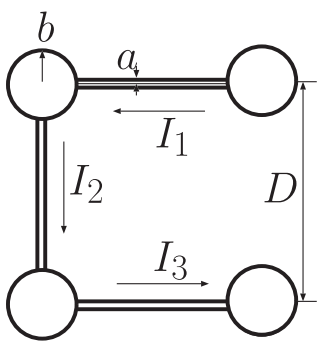

Fig. 7 Simple example of a meta-atom.

where the matrix $\boldsymbol{K}$ is a mode decomposition matrix. The matrix $\boldsymbol{K}^{-1} \boldsymbol{L}^{-1} \boldsymbol{U}^{T} \boldsymbol{P} \boldsymbol{U} \boldsymbol{K}$ is a diagonalized matrix, and the vector $-\mathrm{j} \omega \boldsymbol{K}^{-1} \boldsymbol{L}^{-1} \hat{\boldsymbol{V}}^{\mathrm{E}}$ on the right-hand side represents the excitation of each mode by an incident field.

As a simple example with multiple modes, we find resonant modes of the simple meta-atom shown in Fig. 7. The example consists of mutually coupled parallel wires, and the spheres are located with strong couplings. Because the structure has three wires, the equivalent circuit model has three modes for the structure. In this case, the matrices in Eq. (32) are given by

$$
\begin{aligned}
& \boldsymbol{U}=\left[\begin{array}{ccc}
1 & 0 & 0 \\
-1 & 1 & 0 \\
0 & -1 & 1 \\
0 & 0 & -1
\end{array}\right], \quad \boldsymbol{L}=\left[\begin{array}{ccc}
L_{\mathrm{s}} & 0 & L_{\mathrm{m}} \\
0 & L_{\mathrm{s}} & 0 \\
L_{\mathrm{m}} & 0 & L_{\mathrm{s}}
\end{array}\right], \\
& \boldsymbol{P}=\frac{1}{4 \pi \epsilon_{0}}\left[\begin{array}{cccc}
\frac{1}{b} & \frac{1}{D} & \frac{1}{\sqrt{2} D} & \frac{1}{D} \\
\frac{1}{D} & \frac{1}{b} & \frac{1}{D} & \frac{1}{\sqrt{2} D} \\
\frac{1}{\sqrt{2} D} & \frac{1}{D} & \frac{1}{b} & \frac{1}{D} \\
\frac{1}{D} & \frac{1}{\sqrt{2} D} & \frac{1}{D} & \frac{1}{b}
\end{array}\right], \\
& L_{\mathrm{s}}=\frac{\mu_{0}}{4 \pi}\left\{l\left(\log \frac{D^{2}-l^{2}}{a^{2}}-1\right)+D\left(\log \frac{D+l}{D-l}-1\right)\right\}, \\
& L_{\mathrm{m}}=\frac{\mu_{0} D}{2 \pi}\{\log (1+\sqrt{2})-\sqrt{2}+1\} .
\end{aligned}
$$

Because the matrix $\boldsymbol{L}^{-1} \boldsymbol{U}^{T} \boldsymbol{P} \boldsymbol{U}$ is a $3 \times 3$ matrix, we obtain three resonant modes. Figure 8 shows the resonant frequencies and current distribution on each wire. Thus, we easily find the resonant modes by the equivalent-circuit model.

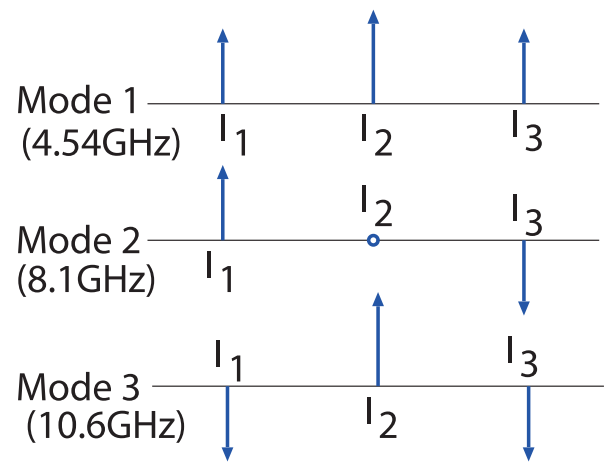

Fig. 8 Three calculated modes of the structure in Fig. 7.

\section{Modeling of Radiation}

\subsection{Radiation Resistance}

The equivalent-circuit model in Eq. (31) does not contain the resistance because we assume the wires and spheres to be perfect conductors. However, the current induced on the wires is attenuated by radiation, even if the ohmic loss is neglected. To estimate the amplitude of the current induced on the wires, we add the radiation loss to the equivalentcircuit model using the radiation resistances that are given with respect to each mode.

We define $\hat{I}_{k}$ by the $k$-th component of the mode current vector $\boldsymbol{K}^{-1} \hat{\boldsymbol{I}}$ in Eq. (35). Then, the radiated power for the $k$ th mode is represented by

$$
P_{k}^{\mathrm{R}}=\frac{1}{2}\left|\hat{I}_{k}\right|^{2} R_{k}^{\mathrm{R}},
$$

where $R_{k}^{\mathrm{R}}$ is the radiation resistance with respect to the $k$-th mode.

In order to calculate the radiated power for each mode, we use a Poynting vector in the far field. We assume that a small structure of the wired spheres exists around the coordinate origin and calculate the far field at an observation point

$$
\xi=(r \sin \theta \cos \varphi, r \sin \theta \sin \phi, r \cos \theta) .
$$

We define the center point of the $n$-th wire as $\left(x_{n}, y_{n}, z_{n}\right)$. Then, the distance between the wire and the observation point is approximated by

$$
r_{n} \simeq r-x_{n} \sin \theta \cos \varphi-y_{n} \sin \theta \sin \varphi-z_{n} \cos \theta .
$$

If we represent the current of the $n$-th wire for the $k$-th mode by $\hat{\boldsymbol{I}}_{n}=\left[\hat{I}_{n}^{\mathrm{X}}, \hat{I}_{n}^{\mathrm{y}}, \hat{I}_{n}^{\mathrm{z}}\right]^{\mathrm{T}}$, the vector potential $\hat{\boldsymbol{A}}$ at the observation point is approximated by

$$
\hat{\boldsymbol{A}} \simeq \frac{\mu_{0} D}{4 \pi r} \sum_{n} \hat{\boldsymbol{I}}_{n} e^{-\mathrm{j} \omega \frac{r_{n}}{c}} .
$$

From Eq. (39) and $\boldsymbol{H}=\frac{1}{\mu_{0}} \boldsymbol{\nabla} \times \boldsymbol{A}$, the far-zone magnetic field is given by 


$$
\begin{gathered}
\hat{H}_{\mathrm{r}}=0, \\
\hat{H}_{\theta}=\frac{\mathrm{j} \omega D}{4 \pi c r} \sum_{n}\left\{-\hat{I}_{n}^{\mathrm{x}} \sin \varphi+\hat{I}_{n}^{\mathrm{y}} \cos \varphi\right\} e^{-\mathrm{j} \omega \frac{r_{n}}{c}}, \\
\hat{H}_{\varphi}=-\frac{\mathrm{j} \omega D}{4 \pi c r} \sum_{n}\left\{\hat{I}_{n}^{\mathrm{x}} \cos \theta \cos \varphi+\hat{I}_{n}^{\mathrm{y}} \cos \theta \sin \varphi\right. \\
\left.-\hat{I}_{n}^{\mathrm{z}} \sin \theta\right\} e^{-\mathrm{j} \omega \frac{r_{n}}{c}} .
\end{gathered}
$$

From the real part of the Poynting vector, we obtain the radiated power $P_{k}^{\mathrm{R}}$ with

$$
P_{k}^{\mathrm{R}}=\frac{1}{2} \sqrt{\frac{\mu_{0}}{\epsilon_{0}}} \int_{0}^{2 \pi} \int_{0}^{\pi}\left(\left|\hat{H}_{\varphi}\right|^{2}+\left|\hat{H}_{\theta}\right|^{2}\right) r^{2} \sin \theta \mathrm{d} \theta \mathrm{d} \varphi .
$$

Then, the radiation resistance is calculated by

$$
R_{k}^{\mathrm{R}}=\frac{\left|\hat{I}_{k}\right|^{2}}{2 P_{k}^{\mathrm{R}}}
$$

To add the damping factor of the $k$-th mode for the radiation, we assume that $\boldsymbol{K}^{-1} \boldsymbol{L}^{-1} \boldsymbol{K}$ is approximately diagonal and introduce a diagonal resistance matrix $\boldsymbol{R}^{\mathrm{R}}$ that has $R_{k}^{\mathrm{R}}$ as the diagonal components. Then, Eq. (35) with the radiation loss is expressed as

$$
\begin{gathered}
\left(-\omega^{2} \mathbf{1}+\mathrm{j} \omega \boldsymbol{K}^{-1} \boldsymbol{L}^{-1} \boldsymbol{K} \boldsymbol{R}^{\mathrm{R}}+\boldsymbol{K}^{-1} \boldsymbol{L}^{-1} \boldsymbol{U}^{T} \boldsymbol{P} \boldsymbol{U} \boldsymbol{K}\right) \boldsymbol{K}^{-1} \hat{\boldsymbol{I}} \\
=-\mathrm{j} \omega \boldsymbol{K}^{-1} \boldsymbol{L}^{-1} \hat{\boldsymbol{V}}^{\mathrm{E}} .
\end{gathered}
$$

This is the complete equivalent-circuit model including the radiation. The damping of the $k$-th mode is estimated by the $k$-th diagonal component of the diagonal matrix $\boldsymbol{K}^{-1} \boldsymbol{L}^{-1} \boldsymbol{K} \boldsymbol{R}^{\mathrm{R}}$.

\subsection{Estimation of Meta-Atoms}

Equation (45) gives not only the resonant frequencies and modes but also the current induced by an external incident field. Here, we show an example of the estimation of the geometric layout for meta-atoms that consist of three wires, as shown in Fig. 9. "Type" in Fig. 9 represents the form of the meta-atoms, and (a), (b), and (c) represent the difference in the excitation by a linearly polarized plane wave. A comparison of the resonant frequencies, external excitations, radiation resistances, and damping of the lowest mode by calculating the ratio to Type I is shown in Fig. 10. The damping is estimated by the diagonal component of the matrix $\boldsymbol{K}^{-1} \boldsymbol{L}^{-1} \boldsymbol{K} \boldsymbol{R}^{\mathrm{R}}$.

Although the difference in the resonant frequencies is small, the orthogonalized wires decreases the inductance, and the resonant frequencies increase. The external excitation is the strongest for Type I and the weakest for Type IVb. The excitation depends on the number of excited wires and the positional relationship. The radiation resistance and damping are the largest for Type I and the smallest for Type IV, and in fact, depend on the coupling of each wire. Thus, the proposed equivalent-circuit model clarifies the differences in the electromagnetic behavior that depends on the

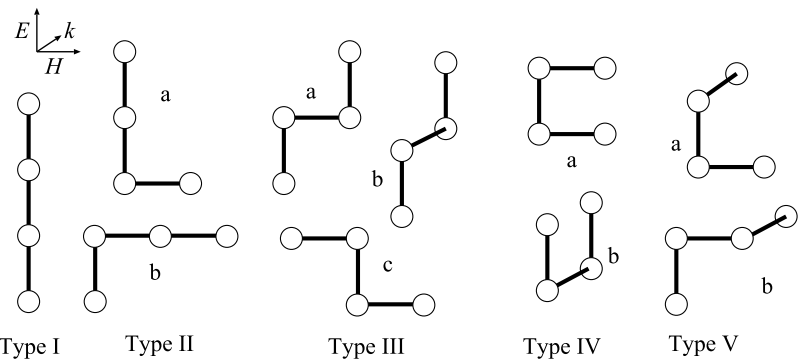

Fig.9 Examples of three-wire structures excited by a linearly polarized plane wave.

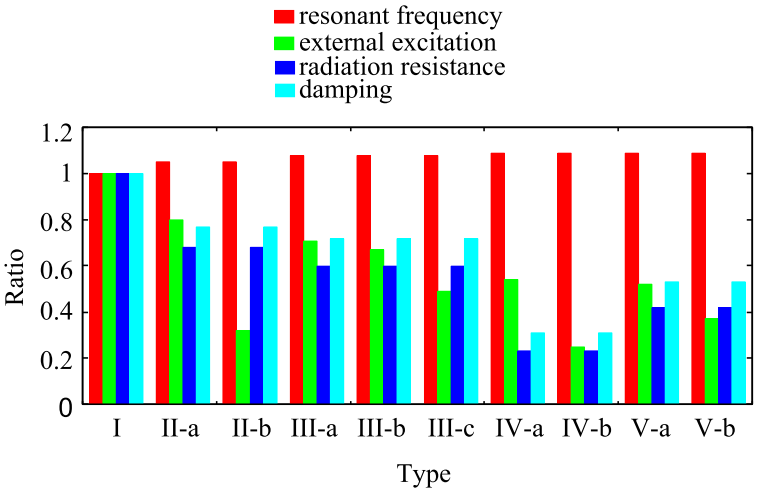

Fig. 10 Comparison of the resonant frequencies, external excitations, radiation resistances, and damping of the lowest mode (using the ratio to Type I).

geometric layout of the meta-atoms.

\subsection{Frequency Characteristics of a Meta-Atom}

We show an example of the frequency characteristics $\hat{I}_{2}(\omega)$ for Type IV-b in Fig. 11. The characteristics include all of the modes from the equivalent-circuit model; however, Mode 2 in Fig. 8 is not excited for this excitation. The results from the electromagnetic field simulation with an FIT are also shown in Fig. 11. It is noted that the results from the proposed model are obtained by using simultaneous linear algebraic equations only with 3 unknowns. Although the equivalent-circuit model is very simple, it provides good approximation. The difference between the resonant frequencies of the proposed model and that of the electromagnetic simulation in Fig. 10 for Mode 3 in Fig. 8 is larger than that for Mode 1. The assumption of uniform current distribution in the wires provides a better approximation for lower frequency based on the condition $l \ll \lambda$ between the wire length $l$ and wavelength $\lambda$ of the incident wave.

\section{Conclusion}

We have proposed a systematic method for deriving an equivalent-circuit model from Maxwell's equations for a structure of wired metallic spheres using the PEEC method under some restrictions. This approach led to a very simple equivalent circuit that has a small degree of freedom and 

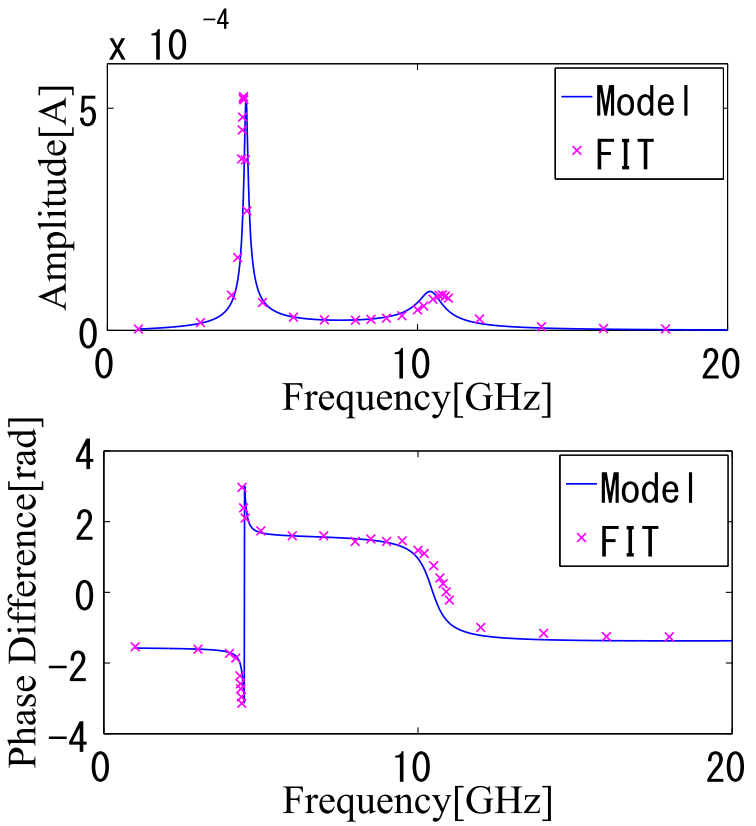

Fig. 11 Comparison of the frequency characteristics of the current $\hat{I}_{2}(\omega)$ for Type IV-b.

the same incidence matrix as the topology of the wires. We also modeled the radiation loss using the radiation resistance with respect to each mode. The equivalent-circuit model provided the resonant frequencies, the resonant modes, the distribution of the current induced in the wires by an external excitation, and so on. Although the model was obtained by a very coarse discretization, it provides a good agreement with the electromagnetic simulation.

\section{Acknowledgements}

This research was supported in part by Grants-in-Aid for scientific Research (Grant Nos. 22109004 and 15K06063).

\section{References}

[1] N. Engheta, "Circuits with light at nanoscales: Optical nanocircuits inspired by metamaterials," Science, vol.317, no.5845, pp.1698-1702, Sept. 2007.

[2] J.D. Baena, J. Bonache, F. Martín, R.M. Sillero, F. Falcone, T. Lopetegi, M.A.G. Laso, J. G-García, I. Gil, M.F. Portillo, and M. Sorolla, "Equivalent-circuit models for split-ring resonators and complementary split-ring resonators coupled to planar transmission lines," IEEE Trans. Microw. Theory Techn., vol.53, no.4, pp.1451-1461, April 2005.

[3] F. Bilotti, A. Toscano, L. Vegni, K. Aydin, K.B. Alici, and E. Ozbay, "Equivalent-circuit models for the design of metamaterials based on artificial magnetic inclusions," IEEE Trans. Microw. Theory Techn., vol.55, no.12, pp.2865-2873, Dec. 2007.

[4] M. Shamonin, E. Shamonina, V. Kalinin, and L. Solymar, "Properties of a metamaterial element: Analytical solutions and numerical simulations for a singly split double ring," J. Appl. Phys., vol.95, no.7, pp.3778-3784, April 2004.

[5] A. Alù and S. Maslovski, "Power relations and a consistent analytic model for receiving wire antennas," IEEE Trans. Antennas Propag., vol.58, no.5, pp.1436-1448, May 2010.
[6] I. Liberal and R.W. Ziolkowski, "Analytical and equivalent circuit models to elucidate power balance in scattering problems," IEEE Trans. Antennas Propag., vol.61, no.5, pp.2714-2726, May 2013.

[7] A.E. Ruehli and A.C. Cangellaris, "Progress in the methodologies for the electrical modeling of interconnects and electronic packages," Proc. IEEE, vol.89, no.5, pp.740-771, May 2001.

[8] A.E. Ruehli, "Equivalent circuit models for three-dimensional multiconductor systems," IEEE Trans. Microw. Theory Techn., vol.22, no.3, pp.216-221, March 1974.

[9] A.E. Ruehli and H. Heeb, "Circuit models for three-dimensional geometries including dielectrics," IEEE Trans. Microw. Theory Techn., vol.40, no.7, pp.1507-1516, July 1992.

[10] M.E. Verbeek, "Partial element equivalent circuit (PEEC) models for on-chip passives and interconnects," Int. J. Numer. Model, vol.17, no.1, pp.61-84, 2004.

[11] K. Yoshida, T. Hisakado, and O. Wada, "Resonant mode analysis of metamaterials composed of conducting spheres and wires by equivalent circuit model," Proc. Metamaterials 2012, pp.140-142, Oct. 2012.

[12] T. Hisakado, K. Yoshida, and O. Wada, "Equivalent circuit model with external coupling for metamaterials composed of wired metallic spheres," Proc. Metamaterials 2013, pp.499-501, Sept. 2013.

[13] R. Harrington, Field computation by moment methods, Macmillan, New York, 1968.

[14] A. Sanada, "Characteristics of an isotropic 3-dimensional lefthanded metamaterial composed of wired metallic spheres," IEICE Electron. Express, vol.6, no.11, pp.689-702, 2009.

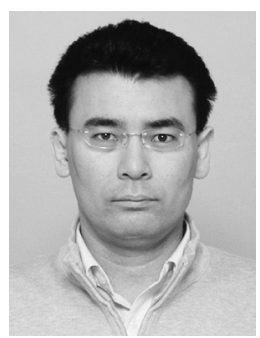

Takashi Hisakado received the B.E. and M.E. degrees in Electrical Engineering II from Kyoto University, in 1993 and 1995, respectively. He received the Dr. degree in Electrical Engineering from Kyoto University, in 1997. He is currently Associate Professor of Department of Electrical Engineering at Kyoto University. His research interests are design of electromagnetic phenomena and power flow. He is a member of IEEJ and IEEE.

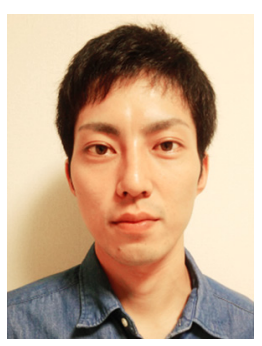

Keisuke Yoshida was born in Fukuoka, Japan, on September 20, 1988. He received his M.E. degree in Electrical Engineering from Kyoto University, Kyoto Japan, in 2013. His research interest is analysis of electromagnetic phenomena in metamaterials by equivalent circuit model.

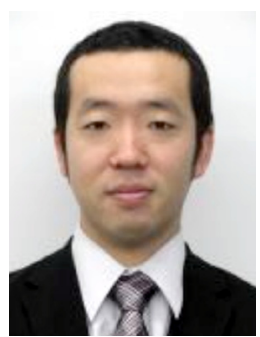

Tohlu Matsushima received his M.E. and $\mathrm{Ph} . \mathrm{D}$. degrees in Communication Network Engineering from Okayama University in 2006 and 2009 , respectively. From 2009, he was an assistant professor in Department of Electrical Engineering at Kyoto University from 2009. His research interest is electromagnetic interference problem. He is a member of IEEE, the Institute of Electrical Engineers of Japan (IEEJ), and the Japan Institute of Electronics Packaging (JIEP). 


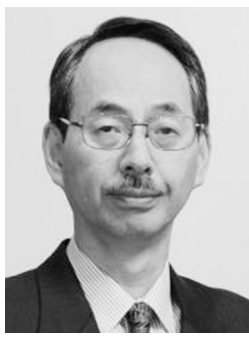

Osami Wada received the B.E., M.E., and Dr. Eng. degrees in Electronics from Kyoto University in 1981, 1983, and 1987, respectively. From 1988 to 2005, he was in the Faculty of Engineering, Okayama University, Japan. In 2005, he became a Full Professor in the Department of Electrical Engineering at Kyoto University. He has been engaged in the study of electromagnetic compatibility (EMC) of electronic circuits and systems, and development of EMC macromodels of integrated circuits. Prof. Wada is a member of IEEE, the Institute of Electrical Engineers of Japan (IEEJ), and the Japan Institute of Electronics Packaging (JIEP). He is a Fellow of the IEICE. 